International Electronic Journal of Algebra

Volume 24 (2018) 91-106

DOI: $10.24330 /$ ieja.440231

\title{
A NOTE ON SIMPLE MODULES OVER QUASI-LOCAL RINGS
}

\author{
Paula A. A. B. Carvalho, Christian Lomp and Patrick F. Smith \\ Received: 28 November 2017; Revised: 10 April 2018; Accepted: 13 April 2018 \\ Communicated by Abdullah Harmancı \\ Dedicated to the memory of Professor John Clark
}

\begin{abstract}
Matlis showed that the injective hull of a simple module over a commutative Noetherian ring is Artinian. In several recent papers, noncommutative Noetherian rings whose injective hulls of simple modules are locally Artinian have been studied. This property had been denoted by property $(\diamond)$. In this paper we investigate, which non-Noetherian semiprimary commutative quasi-local rings $(R, \mathfrak{m})$ satisfy property $(\diamond)$. For quasi-local rings $(R, \mathfrak{m})$ with $\mathfrak{m}^{3}=0$, we prove a characterization of this property in terms of the dual space of $\operatorname{Soc}(R)$. Furthermore, we show that $(R, \mathfrak{m})$ satisfies $(\diamond)$ if and only if its associated graded ring $\operatorname{gr}(R)$ does.

Given a field $F$ and vector spaces $V$ and $W$ and a symmetric bilinear map $\beta: V \times V \rightarrow W$ we consider commutative quasi-local rings of the form $F \times V \times W$, whose product is given by
\end{abstract}

$$
\left(\lambda_{1}, v_{1}, w_{1}\right)\left(\lambda_{2}, v_{2}, w_{2}\right)=\left(\lambda_{1} \lambda_{2}, \lambda_{1} v_{2}+\lambda_{2} v_{1}, \lambda_{1} w_{2}+\lambda_{2} w_{1}+\beta\left(v_{1}, v_{2}\right)\right)
$$

in order to build new examples and to illustrate our theory. In particular we prove that a quasi-local commutative ring with radical cube-zero does not satisfy $(\diamond)$ if and only if it has a factor, whose associated graded ring is of the form $F \times V \times F$ with $V$ infinite dimensional and $\beta$ non-degenerated.

Mathematics Subject Classification (2010): 13E05, 16D50, 13H99, 13D99

Keywords: Quasi-local ring, injective hull, simple module, finiteness condition, commutative ring

\section{Introduction}

The structure and in particular finiteness conditions of injective hulls of simple modules have been widely studied. Rosenberg and Zelinsky's work [15] is one of the earliest studies of finiteness conditions on the injective hull of a simple module. Matlis showed in his seminal paper [13] that any injective hull of a simple module

The first two named authors were partially supported by CMUP (UID/MAT/00144/2013), which is funded by FCT with national (MEC) and European structural funds (FEDER), under the partnership agreement PT2020. 
over a commutative Noetherian module is Artinian. Jans in [11] has termed a ring $R$ to be left co-Noetherian if every simple left $R$-module has an Artinian injective hull. Vamos showed in [20] that a commutative ring $R$ is co-Noetherian if and only if $R_{\mathfrak{m}}$ is Noetherian for any maximal ideal $\mathfrak{m} \in \operatorname{Max}(R)$ - generalising in this way Matlis' result. In connection with the Jacobson Conjecture for non-commutative Noetherian rings, Jategaonkar showed in [12] (see also $[6,17]$ ) that the injective hulls of simple modules are locally Artinian, i.e. any finitely generated submodule is Artinian, provided the ring $R$ is fully bounded Noetherian. We say that a ring $R$ satisfies condition $(\diamond)$ if

Injective hulls of simple left $R$-modules are locally Artinian.

In this paper we study $(\diamond)$ for, not necessarily Noetherian, quasi-local commutative rings $R$ with maximal ideal $\mathfrak{m}$ such that $\mathfrak{m}^{3}=0$. A description of such rings is given in terms of the dual space of $\operatorname{Soc}(R)$ seen as a vector space over $R / \mathfrak{m}$ (Theorem 4.4). Furthermore, we relate property $(\diamond)$ of $(R, \mathfrak{m})$ with its associated graded ring $\operatorname{gr}(R)=R / \mathfrak{m} \oplus \mathfrak{m} / \mathfrak{m}^{2} \oplus \mathfrak{m}^{2}$ in Corollary 4.6. Given a field $F$ and vector spaces $V$ and $W$ and a symmetric bilinear map $\beta: V \times V \rightarrow W$ we consider commutative quasi-local rings of the form $F \times V \times W$, whose product is given by

$$
\left(\lambda_{1}, v_{1}, w_{1}\right)\left(\lambda_{2}, v_{2}, w_{2}\right)=\left(\lambda_{1} \lambda_{2}, \lambda_{1} v_{2}+\lambda_{2} v_{1}, \lambda_{1} w_{2}+\lambda_{2} w_{1}+\beta\left(v_{1}, v_{2}\right)\right)
$$

to build new examples and to illustrate our theory. In particular we prove in Proposition 5.3 that a quasi-local commutative ring with radical cube-zero does not satisfy $(\diamond)$ if and only if it has a factor whose associated graded ring is of the form $F \times V \times F$ with $V$ of infinite dimension and $\beta$ non-degenerate.

\section{Preliminaries}

The following lemma shows that condition $(\diamond)$ is intrinsically linked to Krull's intersection Theorem:

Lemma 2.1. Let $R$ be a (not necessarily commutative) ring with Jacobson radical $J$, such that finitely generated Artinian modules have finite length. If $R$ has property $(\diamond)$, then for any left ideal $I$ of $R$ one has

$$
\bigcap_{n=0}^{\infty}\left(I+J^{n}\right)=I
$$

Proof. Let $I$ be any left ideal of $R$. Then by Birkhoff's theorem $R / I$ embeds into a product of cyclic modules $R / K_{i}$ with essential simple socle, where $I \subseteq K_{i}$ and $\bigcap K_{i}=I$. By hypothesis each of these modules $R / K_{i}$ is Artinian and hence 
has finite length. Thus there exists a number $n_{i} \geq 1$ such that $J^{n_{i}} R / K_{i}=0$, or equivalently $J^{n_{i}} \subseteq K_{i}$. Hence $I+J^{n_{i}} \subseteq K_{i}$ for all $i$ and as the intersection of the $K_{i}$ 's is $I$, we have

$$
I=\bigcap_{i} K_{i} \supseteq \bigcap_{i}\left(I+J^{n_{i}}\right) \supseteq \bigcap_{n}\left(I+J^{n}\right) \supseteq I .
$$

Remark 2.2. (1) Assuming the hypotheses of Lemma 2.1, one can easily adapt the above proof to show that $\bigcap_{n=0}^{\infty}\left(J^{n} M\right)=0$, for any finitely generated left $R$-module $M$. Furthermore, if $M$ is a finitely generated essential extension of a simple left $R$-module, then there exists $n>0$ such that $J^{n} M=0$.

(2) Finitely generated Artinian left $R$-modules have finite length if for example $R$ is left Noetherian or if $R$ is commutative. For the latter case let $M$ be an Artinian module over a commutative ring $R$ generated by $x_{1}, \ldots, x_{k}$. Then

$$
R / \operatorname{Ann}(M) \rightarrow R\left(x_{1}, \ldots, x_{k}\right) \subseteq M^{k}
$$

is an embedding. Since $M^{k}$ is Artinian, $R / \operatorname{Ann}(M)$ is Artinian and by the Hopkins-Levitzki's Theorem R/Ann $(M)$ is Noetherian. As $M$ is finitely generated over $R / \operatorname{Ann}(M), M$ is also Noetherian, i.e. has finite length.

(3) We follow the terminology of commutative ring theory and call a commutative ring $R$ quasi-local if it has a unique maximal ideal $\mathfrak{m}$. A local ring is a commutative Noetherian quasi-local ring. From Lemma 2.1 we see that any commutative quasi-local ring $(R, \mathfrak{m})$, that satisfies $(\diamond)$, is separated in the $\mathfrak{m}$-adic topology. Moreover if $\mathfrak{m}^{n}$ is idempotent, for some $n \geq 1$, then $\mathfrak{m}^{n}=0$.

Recall that a ring $R$ with Jacobson radical $J$ is called semilocal if $R / J$ is semisimple. A semilocal ring with nilpotent Jacobson radical is called semiprimary. The second socle of a module $M$ is the submodule $\operatorname{Soc}_{2}(M)$ of $M$ with $\operatorname{Soc}(M / \operatorname{Soc}(M))=\operatorname{Soc}_{2}(M) / \operatorname{Soc}(M)$. For an ideal $K$ of $R$, denote by $\operatorname{Ann}_{M}(K)$ the set of elements $m \in M$ such that $K m=0$. For a semilocal ring $R$, it is wellknown that $\operatorname{Ann}_{M}(J)=\operatorname{Soc}(M)$ and that $\operatorname{Ann}_{M}\left(J^{2}\right)=\operatorname{Soc}_{2}(M)$. For a left ideal $I$ set $(I: K)=\{r \in R \mid K r \subseteq I\}$.

Proposition 2.3. The following statements are equivalent, for a semiprimary ring $R$ with Jacobson radical $J$.

(a) $R$ has property $(\diamond)$. 
(b) $\operatorname{Soc}_{2}(M)$ has finite length, for any finitely generated left $R$-module $M$ with $\operatorname{Soc}(M)$ finitely generated.

(c) $\left(I: J^{2}\right) / I$ is finitely generated, for any left ideal $I$ of $R$ with $(I: J) / I$ finitely generated.

Proof. Note first that since $R$ is semiprimary, there exists $n \geq 0$ such that $J^{n}=0$. Moreover since $R$ is semilocal, $\operatorname{Soc}(M)=\operatorname{Ann}_{M}(J)=\{m \in M \mid J m=0\}$ for any left $R$-module $M$. In particular any left $R$-module has an essential socle, since any left $R$-module $M$ has a finite socle series:

$$
0=J^{n} M \subseteq J^{n-1} M \subseteq \cdots \subseteq J M \subseteq M .
$$

$(a) \Rightarrow(b)$ If $R$ satisfies $(\diamond)$ then any finitely generated module with finitely generated (essential) socle is Artinian. Hence if $\operatorname{Soc}(M)$ is finitely generated, $M$ must be Artinian and hence $M / \operatorname{Soc}(M)$ is Artinian.

$(b) \Rightarrow(c)$ for $M=R / I$ one has $\operatorname{Soc}(R / I)=(I: J) / I$ as mentioned above. Moreover, $\left(I: J^{2}\right)=((I: J): J)$ and therefore $\left(I: J^{2}\right) /(I: J)=\operatorname{Soc}(R /(I: J))=$ $\operatorname{Soc}(M / \operatorname{Soc}(M))$. Thus the statement follows from $(b)$.

$(c) \Rightarrow(a)$ is clear since if $I$ is a left ideal such that $M=R / I$ is a cyclic essential extension of a simple left $R$-module, then $(I: J) / I$ is cyclic and by assumption $\left(I: J^{2}\right) / I$ is finitely generated. Hence $\left(I: J^{2}\right) / I$ has finite length. Applying our hypothesis to $I^{\prime}=(I: J)$, we can conclude that $\left(I: J^{3}\right) / I$ has finite length. Continuing we have also that $R / I=\left(I: J^{n}\right) / I$ has finite length.

A sufficient condition for a ring to satisfy $(\diamond)$ is given by the following lemma.

Lemma 2.4. Any ring $R$, with $R / \operatorname{Soc}\left({ }_{R} R\right)$ being left Artinian, satisfies ( $\left.\diamond\right)$.

Proof. Suppose $I \subset K \subseteq R$ are left ideals such that $K / I$ is a simple left $R$-module and essential in $R / I$. If $\operatorname{Soc}\left({ }_{R} R\right) \subseteq I$, then $R / I$ is a factor of $R / \operatorname{Soc}\left({ }_{R} R\right)$ and hence Artinian. If $\operatorname{Soc}\left({ }_{R} R\right) \nsubseteq I$, then $\left(\operatorname{Soc}\left({ }_{R} R\right)+I\right) / I$ is a semisimple submodule of $R / I$ and hence must equal $K / I$, i.e. $\operatorname{Soc}\left({ }_{R} R\right)+I=K$. As a quotient of $R / \operatorname{Soc}\left({ }_{R} R\right)$, the module $R / K=R /\left(\operatorname{Soc}\left({ }_{R} R\right)+I\right)$ is Artinian and so is $R / I$.

Clearly it is not necessary for a ring $R$ with $(\diamond)$ to satisfy $R / \operatorname{Soc}\left({ }_{R} R\right)$ being Artinian. Moreover, Example 5.2 shows that there are commutative rings $R$ such that $R / \operatorname{Soc}(R)$ satisfies $(\diamond)$, but $R$ does not.

In recent papers $[2,3,4,5,8,9,14,16]$, several non-commutative Noetherian rings have been shown to satisfy $(\diamond)$. In this note we intend to study condition $(\diamond)$ for commutative not necessarily Noetherian rings. 


\section{Local-global argument}

Jans in [11] defined a ring $R$ to be left co-Noetherian if for every simple left $R$ module its injective hull is Artinian. Vamos has shown in [20] that a commutative ring $R$ is co-Noetherian if and only if $R_{\mathfrak{m}}$ is Noetherian for all $\mathfrak{m} \in \operatorname{Max}(R)$.

The following lemma shows the relation between co-Noetherianess and condition $(\diamond)$ for commutative quasi-local rings. The proof follows the ideas of $[19$, Theorem $1.8]$.

Lemma 3.1. The following statements are equivalent for a commutative quasi-local ring $R$ with maximal ideal $\mathfrak{m}$.

(a) $R$ is Noetherian.

(b) $R$ is co-Noetherian.

(c) $R$ satisfies $(\diamond)$ and $\mathfrak{m} / \mathfrak{m}^{2}$ is finitely generated.

(d) $R$ satisfies $\bigcap_{n=0}^{\infty}\left(I+\mathfrak{m}^{n}\right)=I$ for all (finitely generated) ideals $I$ of $R$ and $\mathfrak{m} / \mathfrak{m}^{2}$ is finitely generated.

Proof. $(a) \Leftrightarrow(b)$ follows from Vamos' result [20, Theorem 2].

$(a) \Rightarrow(c)$ is clear and $(c) \Rightarrow(d)$ follows from Lemma 2.1.

$(d) \Rightarrow(a)$ There exists a finitely generated ideal $B$ of $R$ such that $\mathfrak{m}=B+\mathfrak{m}^{2}$.

Then

$$
\mathfrak{m}=B+\mathfrak{m}^{2}=B+\left(B+\mathfrak{m}^{2}\right)^{2} \subseteq B+\mathfrak{m}^{3} \subseteq \mathfrak{m},
$$

and in general $\mathfrak{m}=B+\mathfrak{m}^{n}$ for every positive integer $n$. It follows from $(d)$ that $\mathfrak{m}=B$ and hence $\mathfrak{m}$ is finitely generated. Suppose that $R$ is not Noetherian. Let $Q$ be maximal among the ideals $C$ of $R$ such that $C$ is not finitely generated. Then $Q$ is a prime ideal of $R$ by a standard argument (see [7, Theorem 2]). Clearly $Q \neq \mathfrak{m}$. Let $p \in \mathfrak{m}$ with $p \notin Q$. By the choice of $Q$ the ideal $Q+R p$ is finitely generated, say

$$
Q+R p=R\left(q_{1}+r_{1} p\right)+\cdots+R\left(q_{k}+r_{k} p\right)
$$

for some positive integer $k, q_{i} \in Q(1 \leq i \leq k), r_{i} \in R(1 \leq i \leq k)$. Let $D=$ $R q_{1}+\cdots+R q_{k} \subseteq Q$. Let $q \in Q \backslash D$. Then by equation (1) there exist $s_{1}, \ldots, s_{k} \in R$ and $d \in D$ such that $q-d=\left(s_{1} r_{1}+\cdots+s_{k} r_{k}\right) p \in Q$. Since $Q$ is prime and $p \notin Q$, $\left(s_{1} r_{1}+\cdots+s_{k} r_{k}\right) \in Q$, i.e. $Q=D+Q p$. Now $Q=D+Q p^{t}$ for every positive integer $t$ and hence

$$
Q=\bigcap_{s=1}^{\infty}\left(D+Q p^{s}\right) \subseteq \bigcap_{s=1}^{\infty}\left(D+\mathfrak{m}^{s}\right)=D .
$$

It follows that $Q=D$ and hence $Q$ is finitely generated, a contradiction. Thus $R$ is Noetherian. 
The diamond condition is equivalent to the condition that any injective hull of a simple $R$-module is locally Artinian. By Remark 2.2, a commutative ring satisfies $(\diamond)$ if and only if any injective hull $E$ of a simple $R$-module is locally of finite length, i.e. any finitely generated submodule of $E$ has finite length.

The last result of this section gives us a local-global argument for condition $(\diamond)$ similar to the one of Vamos for co-Noetherian rings. We start by restating a well-known result that can be found for example in [18, Proposition 5.6].

Proposition 3.2. Let $R$ be commutative ring, $\mathfrak{m}$ a maximal ideal of $R$ and denote by $R_{\mathfrak{m}}$ the localization of $R$ at $\mathfrak{m}$. Then the injective hull $E=E(R / \mathfrak{m})$ of $R / \mathfrak{m}$ as $R$-module is also the injective hull of $R_{\mathfrak{m}} / \mathfrak{m} R_{\mathfrak{m}}$ as $R_{\mathfrak{m}}$-module.

Making use of Proposition 3.2 we can now prove the

Theorem 3.3. A commutative ring $R$ satisfies $(\diamond)$ if and only if $R_{\mathfrak{m}}$ satisfies $(\diamond)$, for all $\mathfrak{m} \in \operatorname{Max}(R)$.

For the proof of Theorem 3.3 we need the following two lemmas. In what follows $\operatorname{Ann}_{S}(-)$ denotes the annihilator of an $S$-module.

Lemma 3.4. Let $R$ be a commutative ring satisfying $(\diamond), \mathfrak{m}$ a maximal ideal of $R$ and $x \in E(R / \mathfrak{m}) \backslash\{0\}$. Then there exists $k \geq 1$ such that $\mathfrak{m}^{k} x=0$.

Proof. Since $R$ satisfies $(\diamond), R x$ has a finite composition series (see Remark 2.2(2)). Hence there exist maximal ideals $\mathfrak{m}_{1}, \ldots, \mathfrak{m}_{n}$ of $R$ such that $\operatorname{Ann}_{R}(x) \subseteq \mathfrak{m}_{i}$, for all $i$ and

$$
\mathfrak{m}_{1} \mathfrak{m}_{2} \cdots \mathfrak{m}_{n} \subseteq \operatorname{Ann}_{R}(x) .
$$

Write $\mathfrak{m}^{k} I=\mathfrak{m}_{1} \mathfrak{m}_{2} \cdots \mathfrak{m}_{n}$ for some $k \geq 0$ and $I$ the product of maximal ideals $\mathfrak{m}_{i} \neq \mathfrak{m}$. Now since $R_{\mathfrak{m}} \mathfrak{m}_{i}=R_{\mathfrak{m}}$ if $\mathfrak{m} \neq \mathfrak{m}_{i}$, it follows

$$
R_{\mathfrak{m}} \mathfrak{m}^{k}=R_{\mathfrak{m}} \mathfrak{m}^{k} I \subseteq R_{\mathfrak{m}} \operatorname{Ann}_{R}(x)=\operatorname{Ann}_{R_{\mathfrak{m}}}\left(R_{\mathfrak{m}} x\right),
$$

where $\operatorname{Ann}_{R_{\mathfrak{m}}}\left(R_{\mathfrak{m}} x\right)=R_{\mathfrak{m}} \operatorname{Ann}_{R}(x)$ follows from [1, Proposition 3.14]. As $x \neq 0$, $k \geq 1$ and the result follows.

Lemma 3.5. Let $R$ be a commutative ring, $\mathfrak{m}$ a maximal ideal of $R, R_{\mathfrak{m}}$ the localization of $R$ at $\mathfrak{m}$ and $x \in E(R / \mathfrak{m}) \backslash\{0\}$. If there exists $k \geq 1$ such that $\mathfrak{m}^{k} x=0$, then $R_{\mathfrak{m}} x=R x$ and the $R$-submodules lattice of $R x$ coincides with the $R_{\mathfrak{m}}$-submodule lattice of $R_{\mathfrak{m}} x$.

Proof. For any $a \in R \backslash \mathfrak{m}$ we have $R=R a+\mathfrak{m}$, which implies also $R=R a+\mathfrak{m}^{k}$. Thus there exists $b \in R$ such that $1-a b \in \mathfrak{m}^{k}$, i.e. $x=a b x$. Hence $a^{-1} x=b x \in R x$ 
shows that $R_{\mathfrak{m}} x=R x$. We have just shown that any principal $R$-submodule of $E=E(R / \mathfrak{m})$ is also a principal $R_{\mathfrak{m}}$-submodule of $E$. Hence the $R$-submodule generated by any set of elements of $E$ coincides with the $R_{\mathfrak{m}}$-submodule generated by that set.

Proof of Theorem 3.3. Let $\mathfrak{m} \in \operatorname{Max}(R)$. By Proposition 3.2, $E=E(R / \mathfrak{m})$ is also the injective hull of the unique simple $R_{\mathfrak{m}}$-module $R_{\mathfrak{m}} / \mathfrak{m} R_{\mathfrak{m}}$.

Assume that $R$ satisfies $(\diamond)$. For $x \in E \backslash\{0\}, R x$ is an Artinian $R$-module and by Lemmas 3.4 and 3.5 also $R_{\mathfrak{m}} x=R x$ is Artinian as $R_{\mathfrak{m}}$-module, so $R_{\mathfrak{m}}$ satisfies $(\diamond)$.

Now assume that $R_{\mathfrak{m}}$ satisfies $(\diamond)$. By hypothesis $E$ is a locally Artinian $R_{\mathfrak{m}}$ module and hence has finite length by Remark 2.2. Hence there exist $k \geq 1$ such that $\left(\mathfrak{m} R_{\mathfrak{m}}\right)^{k} x=0$, for any $x \in E$. In particular $\mathfrak{m}^{k} x=0$ and by Lemma 3.5 it follows that $R x=R_{\mathfrak{m}} x$ has finite length as $R$-module.

Theorem 3.3 shows that in order to characterize commutative rings satisfying $(\diamond)$, we need only to focus on quasi-local commutative rings.

\section{Commutative semiprimary quasi-local rings}

By Lemma 3.1, a quasi-local commutative ring is co-Noetherian if and only if it is Noetherian. Recall, that an ideal $I$ of a ring $R$ is called subdirectly irreducible if $R / I$ has an essential simple socle. Clearly a ring $R$ satisfies $(\diamond)$ if and only if $R / I$ is Artinian, for all subdirectly irreducible ideals $I$ of $R$.

4.1. Commutative quasi-local rings with square-zero maximal ideal. Given any vector space $V$ over a field $F$, the trivial extension (or idealization) is defined on the vector space $R=F \times V$ with multiplication given by $(a, v)(b, w)=(a b, a w+v b)$, for all $a, b \in F$ and $v, w \in V$. Any such trivial extension $R$ is a commutative quasilocal ring that satisfies $(\diamond)$. However $R$ is Noetherian if and only if $V$ is finite dimensional.

Lemma 4.1. Any commutative quasi-local ring with square-zero maximal ideal satisfies $(\diamond)$.

Proof. Let $(R, \mathfrak{m})$ be a commutative quasi-local ring with $\mathfrak{m}^{2}=0$. Since $\mathfrak{m}$ is a vector space over $R / \mathfrak{m}$, it is semisimple. Let $K$ be any subdirectly irreducible ideal of $R$. If $K=\mathfrak{m}$, then $R / \mathfrak{m}$ is simple. So assume $K \subset \mathfrak{m}$. Then there exists a complement $L$ such that $\mathfrak{m}=L \oplus K$ and $\operatorname{Soc}(R / K)=\mathfrak{m} / K \simeq L$ is simple.

$$
0 \longrightarrow \mathfrak{m} / K \longrightarrow R / K \longrightarrow R / \mathfrak{m} \longrightarrow 0
$$


is a short exact sequence. Hence $R / K$ has length 2 .

4.2. Commutative quasi-local rings with cube-zero maximal ideal. In this section we will characterize commutative quasi-local rings $(R, \mathfrak{m})$ with $\mathfrak{m}^{3}=0$ that satisfy $(\diamond)$. Recall that $\operatorname{Soc}(R)=\operatorname{Ann}(\mathfrak{m})=\{r \in R: r \mathfrak{m}=0\}$. Hence $\mathfrak{m}^{2} \subseteq \operatorname{Soc}(R)$. We start with a simple observation.

Lemma 4.2. Let $(R, \mathfrak{m})$ be a commutative quasi-local ring with $\mathfrak{m}^{3}=0$.

(1) If $\mathfrak{m} / \operatorname{Soc}(R)$ is finitely generated, then $R$ satisfies $(\diamond)$.

(2) If $\operatorname{Soc}(R)$ is finitely generated, then $R$ satisfies $(\diamond)$ if and only if $\mathfrak{m} / \operatorname{Soc}(R)$ is finitely generated.

Proof. (1) If $\mathfrak{m} / \operatorname{Soc}(R)$ is finitely generated, then $R / \operatorname{Soc}(R)$ is $\operatorname{Artinian}$ and by Lemma 2.4, $R$ satisfies $(\diamond)$.

(2) If $\operatorname{Soc}(R)$ is finitely generated and $R$ satisfies $(\diamond)$, then $\operatorname{Soc}_{2}(R)=\mathfrak{m}$ is finitely generated, by Proposition 2.3 and in particular $\mathfrak{m} / \operatorname{Soc}(R)$ is finitely generated. Conversely if $\mathfrak{m} / \operatorname{Soc}(R)$ is finitely generated and $\operatorname{Soc}(R)$ is finitely generated, then $\mathfrak{m}$, and so $R$, has finite length. In particular, $R$ satisfies $(\diamond)$.

The last lemma raises the question, whether the reverse conclusion of (1) holds. That is, whether $\mathfrak{m} / \operatorname{Soc}(R)$ needs to be finitely generated for a commutative quasilocal ring $R$ with $\mathfrak{m}^{3}=0$ and satisfying property $(\diamond)$. As we will see in Example 5.4 , this need not be the case.

Lemma 4.3. Let $(R, \mathfrak{m})$ be a commutative quasi-local ring with residue field $F=$ $R / \mathfrak{m}$. Suppose $\mathfrak{m}^{3}=0$. Then there exists a correspondence between subdirectly irreducible ideals of $R$ that do not contain $\operatorname{Soc}(R)$ and non-zero linear maps $f: \operatorname{Soc}(R) \rightarrow F$. Each corresponding pair $(I, f)$ satisfies

$$
\operatorname{Soc}(R) \cap I=\operatorname{ker}(f) \quad \text { and } \quad \operatorname{Soc}(R)+I=V_{f}:=\{a \in \mathfrak{m} \mid f(\mathfrak{m} a)=0\} .
$$

Proof. Let $I$ be a subdirectly irreducible ideal that does not contain $\operatorname{Soc}(R)$, then $\operatorname{Soc}(R / I)=(\operatorname{Soc}(R)+I) / I$ is simple. Thus $\operatorname{Soc}(R)=F x \oplus(\operatorname{Soc}(R) \cap I)$, for a non-zero element $x \in \operatorname{Soc}(R)$. Let $f: \operatorname{Soc}(R) \rightarrow F$ be the linear map such that $\operatorname{ker}(f)=\operatorname{Soc}(R) \cap I$ and $f(x)=1$. Clearly $\operatorname{Soc}(R)+I \subseteq V_{f}$, because

$$
\mathfrak{m}(\operatorname{Soc}(R)+I)=\mathfrak{m} I \subseteq \mathfrak{m}^{2} \cap I \subseteq \operatorname{Soc}(R) \cap I=\operatorname{ker}(f) .
$$

To show that $V_{f}=\operatorname{Soc}(R)+I$ we use the essentiality of $\operatorname{Soc}(R / I)=(\operatorname{Soc}(R)+I) / I$ in $R / I$ as follows: For any $a \in V_{f} \backslash I$, there exists $r \in R$ such that $r a+I$ is a non-zero element of $\operatorname{Soc}(R / I)=(\operatorname{Soc}(R)+I) / I$. Note that $r \notin \mathfrak{m}$ since otherwise 
$f(r a)=0$ and hence $r a \in \operatorname{ker}(f) \subseteq I$. Therefore $r$ is invertible and $a+I=$ $r^{-1} r a+I \in \operatorname{Soc}(R / I)$, i.e. $V_{f}=\operatorname{Soc}(R)+I$.

On the contrary, let $f$ be any non-zero element of $\operatorname{Soc}(R)^{*}=\operatorname{Hom}_{F}(\operatorname{Soc}(R), F)$. Then there exists an element $x \in \operatorname{Soc}(R)$ with $f(x)=1$, such that $\operatorname{Soc}(R)=$ $F x \oplus \operatorname{ker}(f)$. Let $I$ be an ideal of $R$ that contains $\operatorname{ker}(f)$ and that is maximal with respect to $x \notin I$. Thus $I$ is subdirectly irreducible and $\operatorname{Soc}(R / I)=(\operatorname{Soc}(R)+I) / I=$ $(F x \oplus I) / I$ is simple and essential in $R / I$. By construction $\operatorname{ker}(f)=I \cap \operatorname{Soc}(R)$.

Note that $\mathfrak{m}(\operatorname{Soc}(R)+I)=\mathfrak{m} I \subseteq \mathfrak{m}^{2} \cap I \subseteq \operatorname{Soc}(R) \cap I=\operatorname{ker}(f)$, i.e. $\operatorname{Soc}(R)+I \subseteq$ $V_{f}$. To show the reverse inclusion, let $a \in V_{f} \backslash I$, then by essentiality there exists $r \in R$ with $r a=u+v \in \operatorname{Soc}(R)+I, u \in \operatorname{Soc}(R), v \in I$ and $r a \notin I$. If $r \in \mathfrak{m}$, then $r a \in \operatorname{ker}(f) \subseteq I$, contradicting essentiality. Hence $r \notin \mathfrak{m}$ and $a \in \operatorname{Soc}(R)+I$, i.e. $V_{f}=\operatorname{Soc}(R)+I$.

Theorem 4.4. Retain the notation $V_{f}$ from Lemma 4.3. Let $(R, \mathfrak{m})$ be a commutative quasi-local ring with residue field $F$ and $\mathfrak{m}^{3}=0$. Then $R$ satisfies $(\diamond)$ if and only if $\mathfrak{m} / V_{f}$ is finite dimensional over $F$, for any $f \in \operatorname{Soc}(R)^{*}$.

Proof. Suppose that $R$ satisfies $(\diamond)$ and let $f \in \operatorname{Hom}(\operatorname{Soc}(R), F)$. If $f=0$, then $V_{f}=\mathfrak{m}$ and $\mathfrak{m} / V_{f}$ has dimension zero. If $f \neq 0$, then by Lemma 4.3 , there exists a subdirectly irreducible ideal $I$ with $V_{f}=\operatorname{Soc}(R)+I$ and $I$ not containing $\operatorname{Soc}(R)$. As $R$ satisfies $(\diamond), R / I$ is Artinian and as a subquotient $\mathfrak{m} / V_{f}=(\mathfrak{m} / I) /\left(V_{f} / I\right)$ is also Artinian. The $R$-module $\mathfrak{m} / V_{f}$ is semisimple since $\mathfrak{m}^{2} \subseteq V_{f}$. Hence $\mathfrak{m} / V_{f}$ must be finite dimensional as vector space over $F$.

Suppose $\mathfrak{m} / V_{f}$ is finite dimensional for any $f \in \operatorname{Hom}(\operatorname{Soc}(R), F)$. Let $I$ be a subdirectly irreducible ideal of $R$. If $\operatorname{Soc}(R) \subseteq I$, then $R / I$ is an $R / \mathfrak{m}^{2}$-module. Since $R / \mathfrak{m}^{2}$ is a quasi-local ring with square-zero radical, we have by Lemma 4.1 , that $R / \mathfrak{m}^{2}$ satisfies $(\diamond)$. Hence $R / I$ must be Artinian. If $\operatorname{Soc}(R) \nsubseteq I$, then by Lemma 4.3, there exists a non-zero map $f: \operatorname{Soc}(R) \rightarrow F$ such that $\operatorname{Soc}(R)+I=V_{f}$. By hypothesis $\mathfrak{m} / V_{f}$ is finite dimensional and is therefore Artinian as $R$-module. As $R / \mathfrak{m}$ and $V_{f} / I$ are simple modules, also $R / I$ is Artinian, proving that $R / I$ is Artinian for any subdirectly irreducible ideal $I$ of $R$, i.e. $R$ satisfies $(\diamond)$.

Let $(R, \mathfrak{m})$ be any commutative quasi-local ring. The associated graded ring of $R$ with respect to the $\mathfrak{m}$-filtration is the commutative $\operatorname{ring} \operatorname{gr}(R)=\bigoplus_{n \geq 0} \mathfrak{m}^{n} / \mathfrak{m}^{n+1}$ with multiplication given by

$$
\left(a+\mathfrak{m}^{i+1}\right)\left(b+\mathfrak{m}^{j+1}\right)=a b+\mathfrak{m}^{i+j+1}, \quad \forall a \in \mathfrak{m}^{i}, b \in \mathfrak{m}^{j}, \text { and } i, j \geq 0 .
$$

For any ideal $I$ of $R$, the associated graded ideal is $\operatorname{gr}(I)=\bigoplus_{n \geq 0}\left(I \cap \mathfrak{m}^{n}+\right.$ $\left.\mathfrak{m}^{n+1}\right) / \mathfrak{m}^{n+1}$. In particular $\operatorname{gr}(\mathfrak{m})=\bigoplus_{n \geq 1} \mathfrak{m}^{n} / \mathfrak{m}^{n+1}$ is the unique maximal ideal 
of $\operatorname{gr}(R)$. Hence $(\operatorname{gr}(R), \operatorname{gr}(\mathfrak{m}))$ is a commutative quasi-local ring with residue field $F=R / \mathfrak{m}$. Furthermore, $\operatorname{gr}(\operatorname{Soc}(R))$ is contained in $\operatorname{Soc}(\operatorname{gr}(R))$.

In case $\mathfrak{m}^{3}=0$, the associated graded ring of $(R, \mathfrak{m})$ is $\operatorname{gr} R=F \times \mathfrak{m} / \mathfrak{m}^{2} \times \mathfrak{m}^{2}$, where $F=R / \mathfrak{m}$ is the residue field of $R$. For any proper ideal $I$ of $R$ one has

$$
\operatorname{gr}(I)=0 \times\left(I+\mathfrak{m}^{2}\right) / \mathfrak{m}^{2} \times\left(I \cap \mathfrak{m}^{2}\right)
$$

and in particular $\operatorname{gr}(\operatorname{Soc}(R))=0 \times \operatorname{Soc}(R) / \mathfrak{m}^{2} \times \mathfrak{m}^{2}=\operatorname{Soc}(\operatorname{gr}(R))$, because for $\left(0, a+\mathfrak{m}^{2}, b\right) \in \operatorname{Soc}(\operatorname{gr}(R))$ we have that $\left(0, a+\mathfrak{m}^{2}, b\right)\left(0, x+\mathfrak{m}^{2}, 0\right)=(0,0, a x)=$ $(0,0,0)$, for all $x \in \mathfrak{m}$ if and only if $a \in \operatorname{Soc}(R)$. To shorten notation we will write the elements of $\operatorname{gr}(R)$ as $\left(a_{0}, a_{1}, a_{2}\right)$ for $a_{i} \in \mathfrak{m}^{i}$, where the $i$ th component is understood to be modulo $\mathfrak{m}^{i+1}$.

Lemma 4.5. Let $(R, \mathfrak{m})$ be a commutative quasi-local ring with residue field $F$ and $\mathfrak{m}^{3}=0$. For any $f \in \operatorname{Soc}(\operatorname{gr}(R))^{*}=\operatorname{gr}(\operatorname{Soc}(R))^{*}$ there exists $g \in \operatorname{Soc}(R)^{*}$ such that

$$
V_{f}=\{a \in \operatorname{gr}(\mathfrak{m}): f(\operatorname{gr}(\mathfrak{m}) a)=0\}=\operatorname{gr}\left(V_{g}\right),
$$

where $V_{g}$ is the ideal of $R$ defined in Lemma 4.3.

Proof. Let $f: \operatorname{gr}(\operatorname{Soc}(R)) \rightarrow F$ and denote by $\pi: \operatorname{Soc}(R) \rightarrow \mathfrak{m}^{2}$ the projection onto $\mathfrak{m}^{2}$, since $\mathfrak{m}^{2}$ is a direct summand of $\operatorname{Soc}(R)$. Define $g: \operatorname{Soc}(R) \rightarrow F$ by $g(a)=$ $f(0, a, \pi(a))$, for all $a \in \operatorname{Soc}(R)$. Then $(0, x, y) \in V_{f}$ if and only if $f(0,0, t x)=0$, for all $t \in \mathfrak{m}$. Since $t x=\pi(t x) \in \mathfrak{m}^{2}$, the latter is equivalent to $g(t x)=0$ for all $t \in \mathfrak{m}$, i.e. $x \in V_{g}$ (in the ring $R$ ). Hence $V_{f}=\left\{(0, x, y) \in \operatorname{gr}(\mathfrak{m}) \mid x \in V_{g}, y \in\right.$ $\left.\mathfrak{m}^{2}\right\}=\operatorname{gr}\left(V_{g}\right)$.

Corollary 4.6. Let $(R, \mathfrak{m})$ be a quasi-local ring with $\mathfrak{m}^{3}=0$. Then $R$ satisfies $(\diamond)$ if and only if its associated graded ring $\operatorname{gr}(R)$ does.

Proof. If $R$ satisfies $(\diamond)$ and $f: \operatorname{Soc}(\operatorname{gr}(R)) \rightarrow F$ is a non-zero map, then by Lemma 4.5 there exists $g: \operatorname{Soc}(R) \rightarrow F$ such that $V_{f}=\operatorname{gr}\left(V_{g}\right)$. Since $V_{g}$ contains $\operatorname{Soc}(R)$ and hence $\mathfrak{m}^{2}$, we have $V_{f}=\operatorname{gr}\left(V_{g}\right)=0 \times V_{g} / \mathfrak{m}^{2} \times \mathfrak{m}^{2}$. Thus, $\operatorname{gr}(\mathfrak{m}) / V_{f} \simeq \mathfrak{m} / V_{g}$. By Theorem $4.4, \mathfrak{m} / V_{g}$ is finite dimensional as $R$ satisfies $(\diamond)$. Hence $\operatorname{gr}(\mathfrak{m}) / V_{f}$ is finite dimensional for all $f \in \operatorname{Soc}(\operatorname{gr}(R))^{*}$. Again by Theorem 4.4, $\operatorname{gr}(R)$ satisfies $(\diamond)$.

Let $f: \operatorname{Soc}(R) \rightarrow F$ be any non-zero linear map and let $V_{f}=\{a \in \mathfrak{m} \mid f(\mathfrak{m} a)=$ $0\}$. If $\mathfrak{m}^{2} \subseteq \operatorname{ker}(f)$, then $V_{f}=\mathfrak{m}$. If $\mathfrak{m}^{2} \nsubseteq \operatorname{ker}(f)$, then there exists $x \in \mathfrak{m}^{2}$ with $f(x)=1$. Note that $V_{f}^{2} \subseteq \operatorname{ker}(f) \cap \mathfrak{m}^{2}$, hence $I=0 \times\left(V_{f} / \mathfrak{m}^{2}\right) \times\left(\operatorname{ker}(f) \cap \mathfrak{m}^{2}\right)$ is a subdirectly irreducible ideal of $\operatorname{gr}(R)$. To see this note that $E=0 \times 0 \times F x$ is a simple submodule of $\operatorname{gr}(R) / I \simeq F \times \mathfrak{m} / V_{f} \times F x$. We will show that $E$ is 
essential in $\operatorname{gr}(R) / I$. Let $(0, \bar{a}, \bar{b}) \in \operatorname{gr}(R) / I$. If $a \notin V_{f}$, there exists $c \in \mathfrak{m}$ such that $f(a c) \neq 0$ and $a c-f(a c) x \in \operatorname{ker}(f) \cap \mathfrak{m}^{2}$. Hence $(0, \bar{a}, \bar{b})(0, \bar{c}, 0)=(0,0, f(a c) x) \in E$ is non-zero. If $a \in V_{f}$, i.e. $\bar{a}=0$, and $\bar{b} \neq 0$, then $(0,0, \bar{b})$ is a non-zero element of $E$. Hence $E$ is an essential simple submodule of $\operatorname{gr}(R) / I$ and if $\operatorname{gr}(R)$ satisfies $(\diamond)$, the quotient $\operatorname{gr}(R) / I$ and therefore also $\mathfrak{m} / V_{f}$ must be Artinian, thus finite dimensional. By Theorem 4.4, $R$ satisfies $(\diamond)$.

\section{Examples}

Let $(R, \mathfrak{m})$ be a commutative quasi-local ring with $\mathfrak{m}^{3}=0$. The associated graded ring $\operatorname{gr}(R)$ is of the form $\operatorname{gr}(R)=F \oplus V \oplus W$ where $F=R / \mathfrak{m}$ and $V=\mathfrak{m} / \mathfrak{m}^{2}$ and $W=\mathfrak{m}^{2}$ are vector spaces over $F$. Moreover, the multiplication of $R$ induces a symmetric bilinear map $\beta: V \times V \rightarrow W$. Hence $\operatorname{gr}(R)$ is uniquely determined by $(F, V, W, \beta)$ and its multiplication can be identified with the multiplication of a generalised matrix ring. Writing the elements of $S=F \times V \times W$ as 3-tuples $(\lambda, v, w)$ we have that the multiplication is given by

$$
\left(\lambda_{1}, v_{1}, w_{1}\right)\left(\lambda_{2}, v_{2}, w_{2}\right)=\left(\lambda_{1} \lambda_{2}, \lambda_{1} v_{2}+\lambda_{2} v_{1}, \lambda_{1} w_{2}+\lambda_{2} w_{1}+\beta\left(v_{1}, v_{2}\right)\right) .
$$

The units are precisely the elements $(\lambda, v, w)$ with $\lambda \neq 0$ and the unique maximal ideal of $S$ is given by $\operatorname{Jac}(S)=0 \times V \times W$. Let

$$
V_{\beta}^{\perp}=\{a \in V \mid \beta(V, a)=0\},
$$

then $\operatorname{Soc}(S)=0 \times V_{\beta}^{\perp} \times W$, while $\operatorname{Jac}(S)^{2}=0 \times 0 \times \operatorname{Im}(\beta)$.

Recall, that $\beta$ is called non-degenerate or non-singular if $V_{\beta}^{\perp}=0$. In general $\beta$ need not be non-degenerate:

Example 5.1. Let $F$ be any field and $V$ be any vector space over $F$ with countably infinite basis $\left\{v_{0}, v_{1}, v_{2}, \ldots\right\}$. Define the symmetric bilinear form $\beta: V \times V \rightarrow F$ with $\beta\left(v_{0}, v_{0}\right)=1$ and $\beta\left(v_{i}, v_{j}\right)=0$ for any $(i, j) \neq(0,0)$. Then $S=F \times V \times F$ is a commutative quasi-local ring that satisfies $(\diamond)$, because $V_{\beta}^{\perp}=\operatorname{span}\left(v_{i} \mid i>0\right)$ and hence

$$
\operatorname{Jac}(S) / \operatorname{Soc}(S)=(0 \times V \times F) /\left(0 \times V_{\beta}^{\perp} \times F\right) \simeq F
$$

is one-dimensional. By Lemma 4.2, $S$ satisfies $(\diamond)$. Note that $S$ is not Artinian. Moreover, $S=\operatorname{gr}(R)$, where $R=F\left[x_{0}, x_{1}, x_{2} \ldots\right] /\left\langle x_{0}^{3}, x_{i} x_{j} \mid(i, j) \neq(0,0)\right\rangle$.

The bilinear form of the last example was not non-degenerate. Since $0 \times V_{\beta}^{\perp} \times 0$ is always an ideal, we can pass to $F \times V / V_{\beta}^{\perp} \times W$ where the bilinear form $\beta$ is now nondegenerate. The following is a natural example of such a ring with non-degenerate bilinear form: 
Example 5.2. Let $F=\mathbb{R}$ and let $V=C([0,1])$ be the space of continuous real valued functions on $[0,1]$. Set

$$
\langle f, g\rangle=\int_{0}^{1} f(x) g(x) d x, \quad \forall f, g \in C([0,1]) .
$$

Then $\langle\rangle:, V \times V \rightarrow \mathbb{R}$ is a non-degenerate symmetric bilinear form on $V$. Hence, by Lemma 4.2, $R=\mathbb{R} \times V \times \mathbb{R}$ is a commutative quasi-local ring with cube-zero radical, that does not satisfy $(\diamond)$, because its socle $\operatorname{Soc}(R)=0 \times 0 \times \mathbb{R}$ is onedimensional, hence finitely generated, but $\operatorname{Jac}(R) / \operatorname{Soc}(R)$ is infinite dimensional, hence not finitely generated as $R$-module.

These kind of rings must occur as the associated graded ring of a quotient of a commutative quasi-local ring $(R, \mathfrak{m})$ with $\mathfrak{m}^{3}=0$ that does not satisfy $(\diamond)$.

Proposition 5.3. A commutative quasi-local ring $(R, \mathfrak{m})$ with $\mathfrak{m}^{3}=0$ and residue field $F$ does not satisfy $(\diamond)$ if and only if it has a factor $R / I$ whose associated graded ring $\operatorname{gr}(R / I)$ is of the form $F \times V \times F$ with $\operatorname{dim}(V)=\infty$ and non-degenerate bilinear form $\beta: V \times V \rightarrow F$.

Proof. By Theorem 4.4, $R$ does not satisfy $(\diamond)$ if and only if there exists $f \in$ $\operatorname{Soc}(R)^{*}$ such that $\mathfrak{m} / V_{f}$ has infinite dimension. Suppose that $R$ does not satisfy $(\diamond)$ and choose such $f$. Note that $f \neq 0$ since $V_{f} \neq \mathfrak{m}$. By Lemma 4.3 there exists a subdirectly irreducible ideal $I$ of $R$ such that $V_{f}=\operatorname{Soc}(R)+I$ and $\operatorname{ker}(f)=$ $\operatorname{Soc}(R) \cap I$. In particular $(\mathfrak{m} / I)^{2}=V_{f} / I=\operatorname{Soc}(R / I) \simeq F$, because if $\mathfrak{m}^{2} \subseteq I$, then $\mathfrak{m} / I \subseteq \operatorname{Soc}(R / I)=V_{f} / I$ and hence $\mathfrak{m}=V_{f}$, contradicting $V_{f} \neq \mathfrak{m}$. Hence $\mathfrak{m}^{2} \nsubseteq I I$ and $(\mathfrak{m} / I)^{2}=\operatorname{Soc}(R / I)$ as $R / I$ has a simple socle. Moreover, $\operatorname{gr}(R / I)=$ $F \times \mathfrak{m} / V_{f} \times F$, with bilinear form $\beta: \mathfrak{m} / V_{f} \times \mathfrak{m} / V_{f} \rightarrow F$, which is non-degenerate by the definition of $V_{f}$.

On the other hand if $R$ has a factor $R / I$ whose associated graded $\operatorname{ring} \operatorname{gr}(R / I)$ is of the form $F \times V \times F$ with infinite dimensional vector space $V$ and non-degenerate bilinear form $\beta: V \times V \rightarrow F$, then $\operatorname{Soc}(\operatorname{gr}(R / I))=0 \times 0 \times F$ is a simple submodule of $\operatorname{gr}(R / I)$, which is essential since $\beta$ is non-degenerate. As $V$ is infinite dimensional, the semisimple $\operatorname{gr}(R / I)$-module $(0 \times V \times F) /(0 \times 0 \times F)$ is not artinian and hence $\operatorname{gr}(R / I)$ does not satisfy $(\diamond)$. By Corollary $4.6, R / I$ does not satisfy $(\diamond)$ and therefore also $R$ does not.

Let $V=A$ be any unital commutative $F$-algebra. Consider the multiplication of $A$ as a symmetric non-degenerate bilinear map $\mu: A \times A \rightarrow A$ and form the ring $S=F \times A \times A$ as before. In order to apply Theorem 4.4 recall that $\mathfrak{m}=0 \times A \times A$ and $\operatorname{Soc}(S)=0 \times 0 \times A$, as the multiplication of $A$ is non-degenerate. Hence 
elements of $\operatorname{Soc}(S)^{*}$ can be identified with elements of $A^{*}$. For any $f \in A^{*}$ we defined

$$
V_{f}=\{(0, a, b) \in \mathfrak{m} \mid f(A a)=0\}=0 \times I(f) \times A,
$$

where $I(f)$ is the largest ideal of $A$ that is contained in $\operatorname{ker}(f)$. Theorem 4.4 says that $S$ satisfies $(\diamond)$ if and only if $\mathfrak{m} / V_{f} \simeq A / I(f)$ is finite dimensional for any $f \in A^{*}$. From the theory of coalgebras, we borrow the notion of the finite dual $A^{\circ}$ of an algebra, which is the subspace of $A^{*}$ consisting of the elements $f \in A^{*}$ that contain an ideal of finite codimension in their kernel. Hence $S$ satisfies $(\diamond)$ if and only if $A^{\circ}=A^{*}$.

Example 5.4. The trivial extension $A=F \times V$ of a vector space $V$ (see 4.1) is an example of an algebra $A$ satisfying $A^{\circ}=A^{*}$. To see this, note that for any linear subspace $U$ of $A, U \cap V$ is an ideal of $A$. Thus, if $f \in A^{*}$, then $\operatorname{ker}(f) \cap V$ is an ideal of codimension less or equal to 2 and $f \in A^{\circ}$. In particular for such $A, S=$ $F \times A \times A$ satisfies $(\diamond)$. However if $V$ is infinite dimensional, then $\mathfrak{m} / \operatorname{Soc}(S) \simeq V$ is not finitely generated as $S$-module, which shows that the converse of Lemma 4.2(1) does not hold.

However, it might happen that the kernel of an element of $A^{*}$ does not contain an ideal of finite codimension as the following example shows.

Example 5.5. Let $A$ be a commutative unital $F$-algebra with multiplication $\mu$ and $f \in A^{*}$. Note that the composition $\beta=f \circ \mu$ is a non-degenerate bilinear form if and only if $f(A a) \neq 0$, for all non-zero $a \in A$. Or, in other words, $\beta$ is non-degenerate if and only if $\operatorname{ker}(f)$ does not contain any non-zero ideal of $A$. Such a map $f$ can be constructed in case $A=F[x]$ and $F$ has characteristic zero. Suppose $f: F[x] \rightarrow F$ is a linear map and let $0 \neq a=\sum_{i=0}^{n} \lambda_{i} x^{i} \in F[x]$ be such that $f(F[x] a)=0$. For any $m \geq 0$, we have

$$
f\left(x^{m} a\right)=\sum_{i=0}^{n} \lambda_{i} f\left(x^{m+i}\right)=0 .
$$

Thus $v=\left(\lambda_{0}, \lambda_{1}, \ldots, \lambda_{n}\right)$ is in the kernel of the linear map given by the matrix:

$$
B_{n}=\left(\begin{array}{lllll}
f(1) & f(x) & f\left(x^{2}\right) & \cdots & f\left(x^{n}\right) \\
f(x) & f\left(x^{2}\right) & f\left(x^{3}\right) & \cdots & f\left(x^{n+1}\right) \\
f\left(x^{2}\right) & f\left(x^{3}\right) & f\left(x^{4}\right) & \cdots & f\left(x^{n+2}\right) \\
\vdots & \vdots & \vdots & \ddots & \vdots \\
f\left(x^{n}\right) & f\left(x^{n+1}\right) & f\left(x^{n+2}\right) & \cdots & f\left(x^{2 n+1}\right)
\end{array}\right)
$$


In particular $\operatorname{det}\left(B_{n}\right)=0$. Hence if the sequence $\left(f\left(x^{n}\right)\right)_{n \in \mathbb{N}}$ produces a sequence of matrices $\left(B_{n}\right)$ that have all non-zero determinant, then for each $0 \neq a=$ $\sum_{i=0}^{n} \lambda_{i} x^{i} \in A$, there exists $0 \leq m \leq n$ such that $f\left(x^{m} a\right) \neq 0$, i.e. $\beta=f \circ \beta$ is non-degenerate.

Matrices of the form of $B_{n}$ are called Toeplitz or Hankel matrices. A particular example of such a matrix is the Hilbert matrix, which is the matrix

$$
B_{n-1}=\left(\begin{array}{ccccc}
1 & \frac{1}{2} & \frac{1}{3} & \cdots & \frac{1}{n} \\
\frac{1}{2} & \frac{1}{3} & \frac{1}{4} & \cdots & \frac{1}{n+1} \\
\frac{1}{3} & \frac{1}{4} & \frac{1}{5} & \cdots & \frac{1}{n+2} \\
\vdots & \vdots & \vdots & \ddots & \vdots \\
\frac{1}{n} & \frac{1}{n+1} & \frac{1}{n+2} & \cdots & \frac{1}{2 n-1}
\end{array}\right)
$$

in case $F$ has characteristic 0 . In 1894, Hilbert computed that $\operatorname{det}\left(B_{n-1}\right)=\frac{c_{n}^{4}}{c_{2 n}}$, where $c_{n}=\prod_{i=1}^{n-1} i^{n-i}$ (see [10]). Hence if we define $f\left(x^{n}\right)=\frac{1}{n+1}$ for any $n \geq 0$, then the kernel of $f$ does not contain any non-zero ideal and the bilinear form $\beta=f \circ \mu$ is non-degenerate. Hence $S=F \times F[x] \times F[x]$ does not satisfy $(\diamond)$ by Proposition 5.3.

Acknowledgement. The authors would like to thank the referee for his/her careful reading and many suggestions, including the idea behind Theorem 3.3. The authors would also like to thank Miodrag Iovanov for pointing out Example 5.4 and for interesting discussions about an earlier draft of this paper. This paper started with a visit of the last named author to the University of Porto in September 2011. The authors would also like to thank Alveri Sant'Ana for having discussed the results of this paper during his visit to Porto.

\section{References}

[1] M. F. Atiyah and I. G. Macdonald, Introduction to Commutative Algebra, Addison-Wesley Publishing Co., Reading, Mass.-London-Don Mills, Ont., 1969.

[2] K. Brown, P. A. A. B. Carvalho and J. Matczuk, Simple modules and their essential extensions for skew polynomial rings, ArXiv e-prints, (2017), available at 1705.06596 .

[3] P. A. A. B. Carvalho, C. Lomp and D. Pusat-Yilmaz, Injective modules over down-up algebras, Glasg. Math. J., 52(A) (2010), 53-59. 
[4] P. A. A. B. Carvalho and I. M. Musson, Monolithic modules over Noetherian rings, Glasg. Math. J., 53(3) (2011), 683-692.

[5] P. A. A. B. Carvalho, C. Hatipoglu and C. Lomp, Injective hulls of simple modules over differential operator rings, Comm. Algebra, 43(10) (2015), 42214230.

[6] G. Cauchon, Anneaux de polynômes essentiellement bornés, Ring theory (Proc. Antwerp Conf. (NATO Adv. Study Inst.), Univ. Antwerp, Antwerp, 1978), Lecture Notes in Pure and Appl. Math., vol. 51, Dekker, New York, (1979), $27-42$.

[7] I. S. Cohen, Commutative rings with restricted minimum condition, Duke Math. J., 17 (1950), 27-42.

[8] C. Hatipoglu, Stable torsion theories and the injective hulls of simple modules, Int. Electron. J. Algebra, 16 (2014), 89-98.

[9] C. Hatipoglu and C. Lomp, Injective hulls of simple modules over finite dimensional nilpotent complex Lie superalgebras, J. Algebra, 361 (2012), 79-91.

[10] D. Hilbert, Ein Beitrag zur Theorie des Legendre'schen Polynoms, Acta Math., 18(1) (1894), 155-159.

[11] J. P. Jans, On co-Noetherian rings, J. London Math. Soc., 1(2) (1969), 588-590.

[12] A. V. Jategaonkar, Certain injectives are Artinian, Noncommutative ring theory (Internat. Conf., Kent State Univ., Kent Ohio, 1975), Lecture Notes in Math., Vol. 545, Springer, Berlin, (1976), 128-139.

[13] E. Matlis, Injective modules over Noetherian rings, Pacific J. Math., 8 (1958), 511-528.

[14] I. M. Musson, Finitely generated, non-Artinian monolithic modules, New trends in noncommutative algebra, Contemp. Math., 562, Amer. Math. Soc., Providence, RI, (2012), 211-220.

[15] A. Rosenberg and D. Zelinsky, Finiteness of the injective hull, Math. Z., 70 (1958/1959), 372-380.

[16] A. Sant'Ana and R. Vinciguerra, On cyclic essential extensions of simple modules over differential operator rings, ArXiv e-prints, (2017), available at 1704.04970.

[17] W. Schelter, Essential extensions and intersection theorems, Proc. Amer. Math. Soc., 53(2) (1975), 328-330.

[18] D. W. Sharpe and P. Vámos, Injective Modules, Cambridge Tracts in Mathematics and Mathematical Physics, No. 62, Cambridge University Press, London-New York, 1972. 
[19] P. F. Smith, The Artin-Rees property, Paul Dubreil and Marie-Paule Malliavin Algebra Seminar, 34th Year (Paris, 1981), Lecture Notes in Math., 924, Springer, Berlin-New York, (1982), 197-240.

[20] P. Vámos, The dual of the notion of "finitely generated", J. London Math. Soc., 43 (1968), 643-646.

Paula A. A. B. Carvalho and Christian Lomp (Corresponding Author)

Department of Mathematics

Faculty of Science

University of Porto

Rua Campo Alegre, 687

4169-007 Porto, Portugal

e-mails: pbcarval@fc.up.pt (P. A. A. B. Carvalho)

clomp@fc.up.pt (C. Lomp)

Patrick F. Smith

School of Mathematics and Statistics

University of Glasgow

Glasgow G12 8SQ, Scotland

e-mail: patrick.smith@talktalk.net 